\title{
Transformation Based Insertion of On-Line Testing Resources in a High-Level Synthesis Environment
}

\author{
Petros OIKONOMAKOS \\ Mark ZWOLINSKI \\ Electronic Systems Design Group, Department of Electronics and Computer Science \\ University of Southampton, Southampton SO17 1BJ, UK \\ \{po00r,mz\}@ecs.soton.ac.uk
}

\begin{abstract}
On-line testability is essential in designs with high reliability requirements. High-level synthesis reduces time-to-market and enables efficient design space exploration. In our work, we implement on-line testable designs in a high-level synthesis context. We refer to our new technique (inversion testing) and exploit its features, in an attempt to reduce hardware penalties.
\end{abstract}

\section{Introduction}

Many attempts have been made to utilize components' idle time in synthesized designs, in order to provide selfchecking properties. When no more idle time is available, such techniques turn to either time or hardware redundancy. In our approach, we exploit an existing high-level synthesis system, such that the choice between time or hardware redundancy is effectively up to the designer.

\section{Existing synthesis system}

We have been using the MOODS High-Level Synthesis Suite [1]. It is a cost-function driven, transformation-based system, and its resulting designs consist of a data path and a controller, joint together by glue logic.

When MOODS is first invoked, the behavioural HDL description is parsed, and an initial, naïve implementation of the design is formulated. Consequently, scheduling and allocation transforms are selected (from a set of available transforms), their validity is tested, their impact on the design parametres is evaluated (through a cost function which reflects designer requirements), and some of them are applied, thus optimizing the design.

\section{Proposed techniques and progress so far}

The well-known algorithmic duplication testing scheme forms the basis of our self-checking technique. We also utilize the inversion testing scheme (figure 1), which we proposed in [2]. Experiments show that

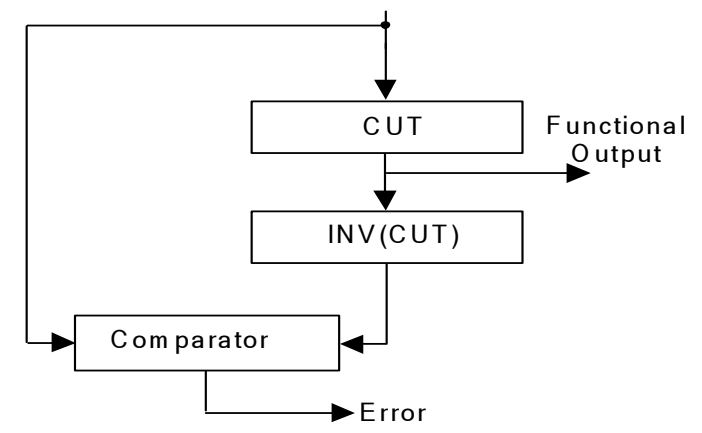

Figure 1. Inversion Testing

inversion can be an interesting supplement to duplication.

In order to integrate our techniques within MOODS, we supplement the existing set of transforms with a few duplicating / inverting and comparing transforms. Test resource insertion is still semi-automatic, in the sense that, while no HDL modification is needed, some user interaction through the MOODS GUI is required. We are working towards full automation, by supplementing the system cost function with a metric for on-line testability.

\section{Conclusion}

The contribution of this research work can be summarized in the following three points.

Test resources are inserted automatically at the behavioural level. No modification of HDL description is required. Thus, designs of substantial (industrial) sizes can easily be made on-line testable (for the first time).

The inversion testing technique provides additional flexibility towards minimizing overheads.

On-line testability emerges as an additional dimension in the design space. We expect this idea to be useful in other contexts as well.

\section{References}

[1] A.C. Williams, "A Behavioural VHDL synthesis system using data path optimisation", $\mathrm{PhD}$ Thesis, University of Southampton, 1997.

[2] P. Oikonomakos, M. Zwolinski, "Using High-Level Synthesis to Implement On-Line Testability”, IEEE/IEE RealTime Embedded Systems Workshop, London, UK, 2001, pp. 135-138. 